\title{
Cervical spine surgery in patients with rheumatoid arthritis: an appraisal
}

\author{
E R McRorie, P McLoughlin, T Russell, I Beggs, G Nuki, N P Hurst
}

\begin{abstract}
Objectives-To review the outcome of surgery undertaken to stabilise the neck in patients with rheumatoid arthritis performed over a five year period, to compare the results with those of previous reports, and to identify factors that may predict surgical outcome.

Methods-Outcome was assessed at time of discharge from hospital after surgery by review of patients' notes, and at follow up by patient interview, clinical examination, anonymous questionnaire, and cervical spine radiograph. The Ranawat classification of neurological impairment and Steinbrocker functional classification were used.
\end{abstract}

Results-Thirty nine patients underwent 44 procedures; 28 patients were available for review after a mean period of 29.8 months (range 12-65 months). Fourteen patients had preoperative neurological impairment and were available for follow up; 13 returned the questionnaire. Four (29\%) had improved Ranawat class, nine were unchanged, and one had deteriorated. Nine $(69 \%)$ reported a subjective improvement in neurological symptoms by questionnaire, even though the Ranawat class was unchanged in five. Twenty five of the patients reviewed had pain before operation; 21 returned the questionnaire. Pain relief was reported by direct questioning and questionnaire in $76 \%$ and $67 \%$ of patients, respectively. Overall, $67 \%$ felt that surgery had been successful. Surgery was more successful in producing symptomatic relief in patients with neck or radicular pain than in those with neurological deficit, but did prevent progression of neurological symptoms.

Conclusions-Our results are similar to those from other centres. Overall patient satisfaction with surgery was good. Surgery was more likely to produce symptomatic relief in patients with neck or radicular pain before operation than in those with neurological deficit. The greater subjective improvement in neurological symptoms as judged by questionnaire probably reflects the relative insensitivity of the Ranawat classification in detecting change in neurological status; previous reports of poor outcome for patients with neurological symptoms who undergo surgery may in part be a reflection of the insensitivity of this method of assessment. No clear factors emerged which allowed prediction of those patients at greatest risk of operative mortality. In particular, an increased risk of neurological compromise appeared to confer no additional risk of immediate perioperative death. Our data support the suggestion that early surgery to correct symptomatic atlantoaxial subluxation may prevent progression of instability.

(Ann Rheum Dis 1996; 55: 99-104)

Involvement of the cervical spine is common in rheumatoid arthritis (RA); atlantoaxial subluxation, the commonest form of instability, has been reported in $32 \%$ of inpatients with the disease. ${ }^{1}$ Synovitis, bony destruction secondary to erosive disease and osteoporosis, and damage to the supporting soft tissue structures of the neck, crucially the transverse and alar ligaments at the atlantoaxial level, may produce neck instability. Pain, neurological impairment, or even sudden death may result.

We have reviewed the outcome of surgery undertaken to stabilise the neck in Edinburgh over a five year period in patients with $R A$. The purposes of the study were to compare our results with those of previous reports and to try to identify preoperative factors that predicted surgical outcome. We also wished to examine how well informed patients felt they had been about the nature of and indications for surgery, and how successful they felt surgery had been in producing symptom relief.

Previous reviews of neck surgery in RA have found posterior stabilisation to be effective in relieving pain in $60-100 \%$ of patients. ${ }^{2-6}$ Results of surgery performed in patients with neurological deficit are not as encouraging, and may depend on the degree of neurological impairment. ${ }^{4}$ Instability is commonest at the atlantoaxial junction (atlantoaxial subluxation or antlantoaxial impaction (cranial settling)), but may also develop below C2 (subaxial subluxation). The indications for surgical stabilisation are generally considered to be intractable pain and progressive neurological deficit. Neurological assessment may be difficult in the rheumatoid patient, however, in whom clinical signs may be masked by joint contractures and muscle atrophy; a mean delay in diagnosis of cervical myelopathy of 31 weeks was documented in one series. ${ }^{8}$ The role of prophylactic surgery in an asymptomatic individual in whom gross instability is discovered as an incidental finding is contentious, as there is poor correlation between the degree of subluxation seen on plain radiographs and the development of complications. This is partly 
Table 1 Ranawat classification of neurological impairment

\begin{tabular}{ll}
\hline Ranawat class & Neurological status \\
\hline I & No neurological deficit \\
II & Subjective weakness with hyperreflexia and dysaesthesia \\
IIIA & Objective weakness and long tract signs; ambulatory \\
IIIB & Objective weakness and long tract signs; bed or chair bound \\
\hline
\end{tabular}

the result of formation of pannus-a mass of inflammatory tissue not visible on plain radiographs, which can compress the spinal cord. ${ }^{9}$

\section{Patients and methods}

A retrospective survey was performed of all patients with RA who had undergone surgery to stabilise the neck in Edinburgh during the period 1988-92. Patients were identified from a database containing demographic details of all those who had undergone cervical spine surgery during this period. All procedures except one were performed by one surgeon (TR). Patients' case notes were reviewed to obtain demographic details including serological status, details of preoperative symptoms and signs, form of cervical instability, functional status (Steinbrocker), and treatment with corticosteroids. Immediate postoperative outcome at time of discharge from hospital was also assessed from the patients' notes.

Subjects were then invited to attend for clinical review, when functional capability, pain, and neurological symptoms were documented, and a neurological examination was performed. The Ranawat classification of neurological impairment was used (table 1). ${ }^{3}$ Cervical spine radiographs (lateral flexion/ extension, and anteroposterior views) were also taken. Atlantoaxial subluxation was defined as a gap of greater than $3 \mathrm{~mm}$ between the posterior surface of the anterior arch of the atlas and the anterior border of the odontoid peg with the neck in flexion; ${ }^{10}$ subaxial subluxation was said to be present if there was a gap of greater than $3.5 \mathrm{~mm}$ between the posterior borders of adjacent vertebrae; ${ }^{11}$ atlantoaxial impaction was diagnosed if the tip of the odontoid peg protruded more than $4.5 \mathrm{~mm}$ above McGregor's line (a line drawn from the posterior edge of the "hard palate to the most caudal point on the occiput)..$^{12}$ Radiographs were assessed by a consultant radiologist (IB) unaware of the outcome of surgery.

Patients were also given an anonymous questionnaire and prepaid envelope and were asked to assess (anonymously) how well informed they had been about the nature of and indications for surgery, and how effective surgery had been in producing relief of their symptoms. The questionnaire comprised 14 closed questions with rating scales, and one open question.

Table 2 Perioperative deaths-preoperative clinical status

\begin{tabular}{llllllll}
\hline $\begin{array}{c}\text { Age } \\
(y r)\end{array}$ & Sex & Duration of RA & Steroids & Instability & Pain & Ranawat & Steinbrocker \\
\hline 75 & F & 30 years & Yes & SAS & Yes & II & III \\
65 & M & 15 years & No & AAS & Yes & II & III \\
75 & F & 25 years & No & AAS & Yes & II & II \\
\hline
\end{tabular}

$\mathrm{RA}=$ Rheumatoid arthritis; SAS = subaxial subluxation; $\mathrm{AAS}=$ atlantoaxial subluxation.
Thirty nine patients underwent 44 procedures. Twenty seven patients $(69 \%)$ were women. The mean age at time of surgery was 60.9 years (range 38-78 years) and mean duration of RA was 19.6 years (range 4-40 years). Twenty six patients $(67 \%)$ were known to be seropositive and 21 (54\%) were receiving corticosteroids at the time of surgery. Twenty eight $(72 \%)$ of the 39 patients were available for follow up after a mean period of 29.8 months (range 12-65 months). Of the remaining 11 patients, four died during the period of follow up, two patients could not be contacted, and two had moved from area; there were three perioperative deaths.

\section{Results}

FORMS OF INSTABILITY AND OPERATIVE PROCEDURES

Thirty three operations were performed for atlantoaxial subluxation, eight for subaxial subluxation, and three for composite forms of instability (one patient subaxial subluxation and atlantoaxial impaction, two patients atlantoaxial subluxation and subaxial subluxation). Most patients with atlantoaxial subluxation underwent posterior stabilisation with Ransford loop $^{1314}(n=23)$ or wiring $(n=8$; five with bone graft); 12 patients also underwent transoral resection of dens. Patients with subaxial subluxation underwent decompressive procedures.

\section{POSTOPERATIVE OUTCOME}

Perioperative mortality

Three patients died in the perioperative period (table 2). All were in Ranawat class II before operation, and died 10-24 days after surgery. Two of these patients developed quadraparesis following surgery.

There were four further deaths during the period of follow up, occurring nine, 34,38 , and 44 months after surgery. The cause of death in three patients was a myocardial infarction; the fourth died as a result of bronchopneumonia. Two of these four were in Ranawat class IIIB before operation, one was in Ranawat class I, and the other Ranawat class II.

\section{Surgical outcome}

Four patients underwent further surgery for recurrent instability. Surgery was considered technically unsuccessful in another four patients in whom revision was undertaken within four months of the initial operation (tightening of a Halifax system, revision of a bone graft, repositioning of a Ransford loop, and replacement of a loose Halifax system with a Ransford loop). As these procedures were performed for technical rather than clinical indications, they have not been included in the results outlining symptomatic outcome.

\section{Symptomatic outcome}

Symptomatic outcome (pain and neurological symptoms) was assessed at the time of the 
Table 3 Symptomatic outcome-pain

\begin{tabular}{lll}
\hline $\begin{array}{l}\text { Pain severity compared } \\
\text { with preop status }\end{array}$ & $\begin{array}{l}\text { On discharge } \\
(n=32)\end{array}$ & $\begin{array}{l}\text { At follow up } \\
\left(n=25^{\star}\right)\end{array}$ \\
\hline Improved & 19 & 19 \\
Unchanged & 13 & 5 \\
Worse & 0 & 1 \\
Died & 0 & 3 \\
\hline
\end{tabular}

*24 patients who had pain before operation, plus one patient who developed pain during the period of follow up.

patient's discharge from hospital and at follow up. Two patients were asymptomatic before surgery; both remained so after operation. Previous or current use of corticosteroids had no influence on symptomatic (or radiological) outcome. Symptomatic outcome was also independent of duration of follow up; subjects in whom follow up was greater than two years $(n=14)$ had an outcome similar to that of those in whom follow up was less than two years $(n=14)$.

Pain relief (table 3)-Thirty five patients had neck or radicular pain before operation and, of these, 19 also had neurological deficit. Thirty two survived surgery; pain had improved on discharge from hospital in 19 of them (59\%). Twenty five patients were available at follow up: $19(76 \%)$ continued to derive symptomatic relief. In four of these patients, pain was unchanged when they were discharged from hospital, but had improved at follow up. In one patient, though pain had improved at the time of discharge from hospital, at follow up the pain was no better than before operation. Another patient developed new neck pain during the period of follow up.

Improvement in neurological status - Twenty five patients had neurological impairment before operation (table 4); 22 of them survived surgery. At time of discharge after surgery, five patients $(23 \%)$ had improved Ranawat class, $16(73 \%)$ were unchanged, and one patient $(4 \%)$ had deteriorated (table 5$)$. Fifteen patients

Table 4 Preoperative Ranawat class of patients who survived surgery

\begin{tabular}{ll}
\hline Neurological status before op. & No patients \\
\hline Ranawat I & 19 \\
Ranawat II & 15 \\
Ranawat III & 3 \\
Ranawat IV & 4 \\
\hline
\end{tabular}

Table 5 Outcome of neurological symptoms

\begin{tabular}{lcc}
\hline $\begin{array}{l}\text { Ranawat class compared } \\
\text { with preop status }\end{array}$ & $\begin{array}{l}\text { On discharge } \\
(n=22)\end{array}$ & $\begin{array}{l}\text { At follow up } \\
\left(n=15^{\star}\right)\end{array}$ \\
\hline Improved & 5 & 4 \\
Unchanged & 16 & 10 \\
Worse & 1 & 1 \\
Died & - & 3 \\
\hline
\end{tabular}

^Fourteen patients who had neurological involvement before operation, plus one patient who developed neurological involvement during the period of follow up.

Table 6 Outcome of preoperative neurological symptoms by Ranawat class

\begin{tabular}{|c|c|c|c|c|}
\hline \multirow{2}{*}{$\begin{array}{l}\text { Ranawat class compared } \\
\text { with preop status }\end{array}$} & \multicolumn{2}{|l|}{ Ranawat II } & \multicolumn{2}{|l|}{ Ranawat III } \\
\hline & $\begin{array}{l}\text { On discharge } \\
(n=15)\end{array}$ & $\begin{array}{l}\text { At follow up } \\
(n=11)\end{array}$ & $\begin{array}{l}\text { On discharge } \\
(n=7)\end{array}$ & $\begin{array}{l}\text { At follow up } \\
(n=4)\end{array}$ \\
\hline $\begin{array}{l}\text { Improved } \\
\text { Unchanged } \\
\text { Worse } \\
\text { Died }\end{array}$ & $\begin{array}{r}5 \\
10 \\
0 \\
-\end{array}$ & $\begin{array}{l}3 \\
7 \\
1 \\
1\end{array}$ & $\begin{array}{l}0 \\
6 \\
1 \\
-\end{array}$ & $\begin{array}{l}1 \\
3 \\
0 \\
2\end{array}$ \\
\hline
\end{tabular}

were reviewed at follow up; improvement had been maintained in four $(27 \%)$. Ranawat class was unchanged compared with the preoperative status in another 10 patients $(67 \%)$. One patient $(6 \%)$ developed fresh neurological impairment during the period of follow up.

Patients who had long tract signs before operation (Ranawat class III) showed the least tendency towards improvement after surgery (table 6). On discharge from hospital, six out of seven $(86 \%)$ of these patients were unchanged and one patient was worse. Among subjects in Ranawat class II before operation, five of $15(33 \%)$ had improved on discharge, and the remainder were unchanged.

Any difference in outcome at follow up was less clear and may reflect the deaths that occurred in the most disabled group during the period of follow up. Of the six patients in Ranawat class III before operation, two had died and four were available for review; among these, three were unchanged and one had improved. Among patients in Ranawat II before surgery, three of $11(27 \%)$ survivors remained improved and seven $(64 \%)$ were unchanged at follow up; one patient ( $9 \%$ ) had developed neurological impairment during the period of follow up.

Patients with neck or radicular pain before operation were more likely to derive symptomatic benefit than those with neurological symptoms when assessed on discharge from hospital $\left(p<0.05\right.$, Yates' corrected $\left.\chi^{2}\right)$ and at follow up $\left(\mathrm{p}<0.01\right.$, Yates' corrected $\left.\chi^{2}\right)$.

\section{Functional outcome}

Table 7 shows the preoperative functional status of patients who survived surgery. Two patients changed functional class after surgery: both deteriorated from Steinbrocker class III to IV, as a result of progressive neurological impairment in one, and increasing joint disability in the other.

\section{Radiographic outcome}

Postoperative cervical spine radiographs were available for review in 24 patients; two patients did not wish to undergo further radiography, and in another two patients, relevant radiographs were not obtainable. Mean time since surgery was 31 months.

Seventeen of these patients had atlantoaxial subluxation before operation. Thirteen underwent posterior stabilisation with Ransford loop (five with transoral resection of dens), and four with wiring (three with bone graft). After surgery, eight patients had an atlantoaxial distance of greater than $3 \mathrm{~mm}$ (mean $8 \mathrm{~mm}$ ) that was stable on flexion/extension views. In two patients the atlantoaxial distance was

Table 7 Preoperative functional class of patients who Table 7 Preoperat surgery
surved

\begin{tabular}{lc}
\hline Functional class & No patients \\
\hline Steinbrocker I & 3 \\
Steinbrocker II & 12 \\
Steinbrocker III & 18 \\
Steinbrocker IV & 8 \\
\hline
\end{tabular}


reduced to less than $3 \mathrm{~mm}$. Two patients had persisting atlantoaxial subluxation that increased on flexion, one with associated subaxial subluxation, and another two patients had developed atlantoaxial impaction. In the remaining three patients, it was unclear from plain radiographs whether atlantoaxial subluxation was present. Both patients who had subaxial instability before operation had stable flexion/extension radiographs at follow upone with persisting subaxial subluxation. Of the remaining five patients, three had undergone more than one operation (all three for atlantoaxial and subaxial subluxations); the remaining two patients had composite forms of instability dealt with at one procedure (one atlantoaxial and subaxial subluxations; one subaxial subluxation and atlantoaxial impaction). At follow up, two of five patients had persisting atlantoaxial or subaxial subluxation that was stable on flexion/extension. The three other patients had developed or had persisting atlantoaxial impaction. Only one patient developed new instability below the level of surgical fusion during the period of follow up.

Patients with the most complicated forms of instability, therefore, had the least successful radiological outcome as judged by plain radiographs. However, there was no clear correlation with symptomatic or neurological outcome in these patients. Similarly, in patients with isolated atlantoaxial subluxation before operation, clinical outcome appeared to be independent of radiological outcome. There was also no correlation between appearances on preoperative computed tomography or myelography and clinical outcome in patients with isolated altantoaxial subluxation.

\section{Complications}

In addition to the four procedures which were considered technically unsuccessful and were revised, there were two postoperative wound infections, one of which eventually resulted in removal of stabilising wires. One patient developed a posterior fossa hygroma after operation, necessitating surgical evacuation; recovery for this patient was otherwise uneventful. Two patients experienced nasal regurgitation after surgery which resolved with conservative management after several months.

\section{PATIENT QUESTIONNAIRE}

Twenty eight patients received questionnaires and 26 replied (response rate of 93\%), referring to 33 operations (including revision procedures).

Eighty eight percent were of the opinion that they had been given a useful explanation of the nature of their neck instability, and a similar proportion thought that they had received an adequate explanation of what surgery entailed. However, most patients (64\%) stated that they would have found it helpful to have received written information regarding the nature of surgery before their admission to hospital.
Relief of symptoms, as judged by the questionnaire, was good. In particular, relief of neurological symptoms was better than as assessed at clinic review by interview and clinical examination. Sixty nine percent (nine of 13) reported relief of neurological symptoms and $67 \%$ of patients ( 14 of 21 ) reported relief of pain. Overall, $67 \%$ thought surgery had been successful or very successful, $15 \%$ felt worse or much worse, and $18 \%$ felt that surgery had made no difference to their symptoms. Given similar circumstances, $79 \%$ stated that they would undergo the same procedure again, and $73 \%$ would recommend surgery to a friend or relative with a similar problem.

\section{Discussion}

Conservative management of symptomatic cervical spine instability in RA is disappointing, ${ }^{15-17}$ particularly if there is neurological compromise, ${ }^{781819}$ and surgery is often undertaken in an attempt to relieve symptoms and produce neck stability. The surgery involved, however, is technically demanding; poor bone quality may make it difficult to secure stabilising devices, and impaired wound healing, susceptibility to infection, and joint disability may limit postoperative rehabilitation. It would be useful, therefore, to be able to predict which patients were unlikely to benefit from surgery. We have assessed the outcome of surgery on discharge from hospital and at clinic review, in terms of surgical (technical) outcome, symptomatic improvement as judged by clinical impression and anonymous patient questionnaire, change in functional status, and radiographic outcome.

Although there are difficulties when comparing these results with earlier studiesbecause of differences in patient selection, and the procedures performed (including the use of traction before operation)-some broad comparisons can be drawn. The perioperative mortality and postoperative complication rates are comparable to those achieved by others $^{3-7} 1^{14}$ and, as in previous studies, ${ }^{2-6}$ pain relief was achieved in the majority of patients ( $76 \%$ at follow up). We found that surgery was more likely to provide symptomatic pain relief than relief of neurological symptoms present before operation, ${ }^{3}{ }^{4}$ though others have achieved a better outcome in patients with neurological compromise. ${ }^{5-7}$

Patients with the most severe degree of neurological impairment showed the least likelihood of improvement after surgery. ${ }^{4}$ Ranawat class was unchanged or had deteriorated after operation in the seven patients who had long tract signs (Ranawat III), compared with improvement in $33 \%$ of patients in Ranawat class II on discharge from hospital, the remainder being unchanged. At follow up there was little difference between Ranawat groups (Ranawat III $25 \%$ improved, remainder unchanged; Ranawat II $27 \%$ improved, $64 \%$ unchanged, $9 \%$ deteriorated) though this may reflect the deaths that occurred in the most disabled group during the period of follow up. 
No clear factors emerged which allowed one to predict those patients at greatest risk of perioperative death. In particular, increased degree of preoperative neurological impairment appeared to confer no additional risk.

To our knowledge, this is only the second study that has attempted to assess the outcome of neck surgery in RA by means of a satisfaction questionnaire. Patient satisfaction in the earlier study was also good..$^{20}$ In those with pain before operation, a positive response was elicited more often by direct questioning than by questionnaire $(76 \% v 67 \%)$. It is interesting, however, that a greater proportion were of the opinion that neurological symptoms had improved at follow up as judged by questionnaire $(69 \%)$ than as assessed by neurological examination (27\%), which may in part reflect insensitivity of the Ranawat classification in detecting change in neurological status. Other studies have relied solely on clinical examination to assess the success of surgery, and this may lead to an unduly pessimistic impression of the postoperative improvement achieved in patients with neurological compromise, particularly those with less severe neurological impairment. Similarly, the lack of improvement in Steinbrocker class after operation may also reflect the insensitivity of this method of functional assessment. Nevertheless, surgery arrested progression of neurological deficit in $87 \%$ of patients who had neurological compromise before operation and who were available for follow up. The arrest of progression of neurological symptoms, therefore, rather than their reversal, is perhaps a more realistic surgical goal. We would agree with Zoma et al, however that the threshold for performing surgery should be greatest in those with the most severe neurological impairment. ${ }^{4}$ We would also consider intractable neck pain in the absence of neurological impairment to be an important indication for surgery.

The high response rate to the questionnaire may reflect our practice of giving the questionnaire to the patients at the clinic, together with a prepaid envelope, and providing an explanation of the aims of the survey. Although $88 \%$ stated that they had been given a useful explanation of the nature of their neck problem and of what surgery entailed, $64 \%$ would have found a written explanation useful. We are therefore compiling a patient information leaflet on neck instability and its treatment, which will outline the nature of and indications for neck surgery in RA, the possible complications, and the postoperative rehabilitation programme.

It has been proposed that early surgery to correct atlantoaxial subluxation may prevent progression of instability, ${ }^{21}$ and our results would lend support to this suggestion in symptomatic atlantoaxial subluxation. Although the number of patients in this study was comparatively small, those patients with atlantoaxial subluxation who developed further instability showed a trend towards longer duration of preoperative symptoms than patients with atlantoaxial subluxation in whom neck stability was maintained when seen for review (19.3 months $v 11.8$ months), despite a longer follow up period in the latter group (26.8 months $v 39 \cdot 1$ months).

\section{Conclusions}

In this study, surgery to stabilise the neck was more likely to produce symptomatic relief in patients with neck or radicular pain than in those with neurological deficit. Surgery was successful, however, in preventing progression of neurological symptoms. In those patients with neurological impairment before operation, those with the least compromise showed the greatest tendency to improve after surgery, as judged by clinical examination and patient questionnaire.

No clear factors emerged to predict those patients at greatest risk of operative mortality. In particular, an increased degree of neurological compromise appeared to confer no additional risk. Although patient numbers were comparatively small, our data support the suggestion that early surgery to correct symptomatic atlantoaxial subluxation may prevent progression of instability.

P McLoughlin was supported by a grant awarded by the Lothian Medical Audit Committee.

1 Conlon P W, Isdale I C, Rose B S. Rheumatoid arthritis of the cervical spine. An analysis of 333 cases. Ann Rheum Dis 1966; 25: 120-6.

2 Clark G R, Goetz D D, Menezes A H. Arthrodesis of the cervical spine in rheumatoid arthritis. $\mathcal{f}$ Bone foint Surg Am 1989; 71: 381-92.

3 Ranawat C S, O'Leary P, Pellicci P, Tsairis P, Marchisello P, Dorr L. Cervical spine fusion in rheumatoid arthritis. $₹$ Bone foint Surg Am 1979; 61: 1003-10.

4 Zoma A, Sturrock R D, Fisher W D, Freeman P A Hamblen D L. Surgical stabilisation of the rheumatoid cervical spine. F Bone foint Surg Br 1987; 69: 8-12.

5 Crockard H A, Essigman W K, Stevens J M, Pozo J L, Ransford A O, Kendall B E. Surgical treatment of cervical cord compression in rheumatoid arthritis. Ann Rheum Dis 1985; 44: 809-16.

6 Heywood A W B, Learmonth I D, Thomas M. Cervical spine instability in rheumatoid arthritis. $\mathcal{F}$ Bone foint Surg Br 1988; 70: 702-7.

7 Boden S D, Dodge L D, Bohlman H H, Rechtine G R. Rheumatoid arthritis of the cervical spine. A long-term analysis with predictors of paralysis and recovery. $¥$ Bone foint Surg Am 1993; 75: 1282-97.

8 Marks J S, Sharp J. Rheumatoid cervical myelopathy. $Q \mathcal{F}$ Med 1981; 199: 307-19.

9 Milbrink J, Nyman R. Posterior stabilisation of the cervical spine in rheumatoid arthritis: clinical results and magnetic resonance imaging correlation. F Spinal Disorders 1990; 3 308-15.

10 Martel W. The occipito-atlanto-axial joints in rheumatoid arthritis and ankylosing spondylitis. Am $\mathcal{f}$ Roentgenol 1961; 86: 223-40.

11 White A A, Johnson R, Panjabi M M, Southwick W O. Biomechanical analysis in clinical stability of the cervical spine. Clin Orthop 1975; 109: 85-96.

$12 \mathrm{McG}$ regor $M$. The significance of certain measurements of the skull in the diagnosis of basilar impression. $\mathrm{Br} \mathcal{F}$ Radiol 1948; 21: 171-81.

13 Ransford A O, Crockard H A, Pozo J L, Thomas N P, Nelson I W. Craniocervical instability treated by contoured loop fixation. $\mathcal{F}$ Bone foint Surg Br 1986; 68: 173 .

14 Crockard H A, Calder I, Ransford A O. One-stage transoral decompression and posterior fixation in rheumatoid decompression and posterior fixation in rheumatoid
atlanto-axial subluxation. $\mathcal{F}$ Bone foint Surg $\mathrm{Br} 1990$; 72: atlanto- $682-5$.

15 Wilson P D, Dangelmajer R C. The problem of atlantoaxial dislocation in rheumatoid arthritis. $\mathcal{F}$ Bone foint Surg Am 1963; 45: 1780 .

16 Althoff B O, Goldie I F. Cervical collars in rheumatoid atlantoaxial subluxation: a radiographic comparison. Ann Rheum Dis 1980; 39: 485-9. 
17 Johnston R A, Kelly I G. Surgery of the rheumatoid cervical spine. Ann Rheum Dis 1990; 49: 845-50.

18 Meijers K A E, Cats A, Kremer H P H, Lugendij K W, Onrlee G J, Thomeer R T W M. Cervical myelopathy in rheumatoid arthritis. Clin Exp Rheumatol 1984; $239-45$.

19 Mathews J A. Atlantoaxial subluxation in rheumatoid arthritis: a five year follow-up. Ann Rheum Dis 1974; 33: arthritis:
20 Krieg J C, Clark C R, Goetz D D. Cervical spine fusion in rheumatoid arthritis: a long-term follow-up. Yale $\mathcal{F}$ Biol Med 1993; 66: 257-62.

21 Agarwal A K, Peppelman W C, Kraus D R, et al. Recurrence of cervical spine instability in rheumatoid arthritis following previous fusion: can disease progression be prevented by early surgery? 7 Rheumatol 1992; 19: prevented $1364-70$. 